\title{
Cardiac Donor Selection and Management
}

\author{
Gillian Grafton $^{1}$ - Gordan Samoukovic ${ }^{2}$ - Monica M. Colvin ${ }^{1}$
}

Published online: 20 October 2015

(C) Springer International Publishing AG 2015

\begin{abstract}
Heart transplantation remains the gold standard treatment for patients with end-stage heart failure. In the USA, the number of available donors has remained stable despite the steadily increasing need for organs. Optimal donor selection and management are critical to deriving the greatest benefit from this limited resource. Methods to expand the donor pool without compromising long-term outcomes for heart recipients are needed.
\end{abstract}

Keywords Heart transplant $\cdot$ Heart donor · Donor selection . Donor management

\section{Introduction}

The recent advancements in surgical technique, immunosuppressive therapy, and myocardial protection have established cardiac transplantation as the ultimate treatment strategy for end-stage heart failure. The dramatic increase in the number of procedures performed during the late 1980s and early 1990s,

This article is part of the Topical Collection on Thoracic Transplantation

Monica M. Colvin

mmcolvin@med.umich.edu

Gillian Grafton

ggrafton@med.umich.edu

Gordan Samoukovic

samoukov@med.umich.edu

1 Department of Medicine, Cardiovascular Division, University of Michigan, 1500 East Medical Drive, Ann Arbor, MI 48109, USA

2 Department of Surgery, Cardiothoracic Division, University of Michigan, 1500 East Medical Drive, Ann Arbor, MI 48109, USA despite persistent mismatch in the donor/recipient numbers, has been attributed to donor pool expansion to include older donors and donors from remote areas, resulting in longer ischemic times [1]. Despite these factors and the increasing age of recipients, cardiac transplantation is nowadays performed with relatively low morbidity and mortality; according to UNOS database, the 1-year survival at high-volume centers approaches $95 \%$ [2]. Still, there remains a donor shortage and there is resurgence in interest in methods to increase access. Optimal donor selection and management are essential in improving conversion of organs and providing the recipient the best opportunity of a good outcome. Frequently, practice has been dictated by prior experience and prevailing myths regarding what constitutes an optimal donor. Herein, we review the available data for optimal donor selection and management.

\section{Donor Assessment and Evaluation}

\section{General}

In general, deceased cardiac donors are declared dead by neurologic criteria (DNC), previously termed brain dead. Once potential heart donors are identified and the donor has been declared brain dead, thorough assessment of donor characteristics and comorbidities as well as heart function must be performed in a timely manner. Evaluation of cardiac function includes serum cardiac enzyme markers (creatinine phosphokinase-MB fraction and troponin), 12-lead electrocardiogram, echocardiogram, and coronary angiogram when indicated [3]. Although serum troponin levels are commonly measured, the association with recipient outcomes has remained unclear. Troponin is commonly used as a marker to evaluate for graft dysfunction, but mildly elevated levels should not limit donor acceptability when found in 
conjunction with normal graft function and normal coronary anatomy [4]. A complete transthoracic echocardiogram should be performed for evaluation of graft function. Important parameters include global ventricular function, wall motion, left ventricular wall thickness, valvular disease, and septal defects [5]. Left ventricular wall thickness greater than $1.4 \mathrm{~cm}$ has been shown to be associated with increased recipient death [6]. Coronary angiogram is typically recommended in male donors $>40$ years old and in female donors $>45$ years old. Pre-existing coronary artery disease may be associated with a higher incidence of progression and development of angiographic allograft coronary artery disease after transplantation, although this does not appear to affect survival $[7,8]$.

\section{Donor Age}

Acceptable donor age is becoming an increasingly debated topic. Early in the transplant experience, the upper age limit of donor age was 35 years. According to the International Society Heart and Lung Transplantation (ISHLT) Registry, the mean age of heart donors rose from 23 years in 1985 to 35 years from 2006 to 2013 [9]. In general, it is widely accepted that increased donor age is associated with poor recipient outcome. According to Del Rizzo and colleagues, donor age above 50 and ischemic time over $4 \mathrm{~h}$ are associated with increased 1- and 2-year mortality. The effect of increasing donor age is quite dramatic at 1- and 2-year marks, reducing the survival to $50.0 \%$ among those who have received an organ from a donor older than 50 years. Additionally, if the ischemic time was beyond $240 \mathrm{~min}$, the 1-year actuarial survival fell to $16.7 \%$ [10]. A more recent analysis of the UNOS registry, examining the interaction of donor age with ischemic time, and their effect on survival, confirms these early findings. Specifically, among the two older age groups (20-33 and $>33$ years), a statistically significant difference was observed between ischemic time and mortality, suggesting greater tolerance for prolonged ischemic times among younger donors. Increased number and duration of comorbidities are often seen in older donors. Furthermore, changes in coronary endothelial function occur as early as age 35 , suggesting a theoretical risk of graft dysfunction after this age. Nevertheless, there is a growing experience with older donors, particularly in Europe. Use of heart donors 50 years and older appears to be safe but associated with higher incidence of cardiac allograft vasculopathy $[11,12]$. Transplant of older donor hearts into older recipients is associated with increased risk of mortality at 5 years as well [13]; however, the risk associated with no transplant was greater than that associated with transplant with an older donor [14]. Thus, in evaluating the risk of accepting an older donor, factors such as ischemic time and the risk of the recipient must be taken into account.

\section{Donor Sex}

Donor-recipient sex mismatch has been associated with poor outcomes. Prendergast and colleagues demonstrated that sex mismatch was associated with a higher number of rejection episodes and reduced survival in the first year after transplantation [15]. There have been several studies that have identified female donor sex as an independent predictor of mortality [16-20]. On closer evaluation, it appears that the greatest negative impact on survival is for male recipients of female donors $[15,21]$ and men who received organs from male donors have the highest cumulative survival at 5 years [22]. Khush and colleagues found that female donor to male recipient transplant was associated with a $10 \%$ increased risk of mortality and reduced graft survival compared to male donor to male recipient; however, in this analysis of ISHLT Registry data, female donor to female recipient conferred a $10 \%$ survival advantage [22]. There was no significant difference in acute rejection or cardiac allograft vasculopathy when sexmatched and sex-mismatched orthotopic heart transplantation (OHT) recipients were compared. In a single-center study, donor gender had no effect on survival in women or men $<45$ years old; however, female donors conferred a higher risk of mortality in men $\geq 45$ years old [21]. The mechanism underlying disparate outcomes with gender mismatch is unclear and may be related to cardiac size mismatch despite matched weight [23]. Evidence to support non-use of a donor heart for gender mismatch is less than compelling, and therefore, sex mismatch should not be perceived as a strong indication for decline.

\section{Donor Size}

Weight within $20-30 \%$ or a donor-to-recipient weight ratio of $0.8-1.2$ is generally considered acceptable for matching. In the 2007 International Society of Heart and Lung Transplantation annual report, Taylor and colleagues found that decreasing donor-to-recipient body mass index ratio was a significant predictor for 5-year mortality [24]. Patel and colleagues evaluated the United Network for Organ Sharing/ Organ Procurement and Transplantation Network Registry from 1999 to 2007 and found that the 30-day mortality was highest for those with a weight ratio $<0.8$ but this finding was not statistically significant. They also evaluated recipients with a pulmonary vascular resistance $>4$ Woods units and showed a trend towards decreased survival in recipients with low weight ratio [25]. However, there is growing evidence that donor weight may not be the best discriminator of risk after transplant. Reed and colleagues demonstrated that when using weight-based matching, there did not appear to be a difference in survival between underweight, overweight, and best-matched donors. However, when predicted left ventricular (LV) mass was used, a mismatch $>10-15 \%$ below the 
recipient predicted LV mass was associated with reduced survival [23].

Some centers have advocated for using oversized hearts to manage elevated pulmonary arterial pressures in the recipients [26]. More recent studies have not shown similar short-term and long-term mortality from both undersized and oversized donors in recipients with mild to moderate pulmonary arterial hypertension [27].

\section{Ischemia Time}

In general, a cold ischemic time $<240 \mathrm{~min}$ is considered optimal, although the impact of increased ischemic times on outcomes has been unclear [28]. When the impact of donor age on ischemic time was evaluated, there was no association between prolonged ischemic time and survival for donors $<20$ years old. On the contrary, survival was decreased in the 20-33-year-old and in the $\geq 34$-year-old terciles with prolonged ischemic times of 3.5-6.24 and 3.5-5.49 h, respectively, and with extended ischemic times of $\geq 6.25$ and $\geq 5.5 \mathrm{~h}$, respectively [2]. Based on these data, ischemic times appear to have the greatest impact among older donors.

\section{Reasons for Non-use of Hearts}

Decreased donor availability remains a difficult reality for patients requiring heart transplantation. In order to increase the donor pool, there has been significant research to help risk stratify potential donors that may have been discarded in the past. Cardiac allograft use decreased from $56 \%$ in 2002 to $37 \%$ use in 2007 [29]. Risk factors for non-use of donor organs include donor age $\geq 45-50$ years, female sex, cerebrovascular accident (CVA), hypertension, diabetes mellitus, history of cocaine or methamphetamine use, high-inotrope requirement, elevated troponin I, left ventricular ejection fraction $<50 \%$, cardiac arrest, hypernatremia, coronary artery disease, left ventricular regional wall motion abnormalities, and left ventricular hypertrophy (septal or posterior wall thickness $>1.1 \mathrm{~cm}$ ) $[29,30]$. Requirement for vasoactive therapy, positive donor cytomegalovirus, longer graft ischemic time, and lower donor body weight are additional reasons for declining organs [19]. Although these are common reasons that donor hearts are declined, they do not necessarily constitute risk factors for outcomes. Although Smits and colleagues found 12 risk factors for non-use, only ventricular hypertrophy $\geq 13 \mathrm{~mm}$ and age constituted risk factors for 3-year mortality [30]. To better understand reasons for declined organs, Khush and colleges evaluated 1872 potential organ donors in the California Transplant Donor Network (CTDN). Of the total number of potential organ donors, $808(43 \%)$ of the donors were accepted for heart transplantation. Donors that were not accepted for transplant were more likely to be older, were female, and had CVA/stroke as a cause of death. These donors also had a higher incidence of smoking, hypertension, diabetes mellitus, coronary artery disease, as well as a positive troponin assay. Using a multivariable model, the most important predictors for allograft use were donor age, cause of death, left ventricular ejection fraction, and history of hypertension. There was no significant difference in the overall survival for recipients of allografts with these risk factors at 1 year after transplant. Diabetes mellitus was the only donor predictor of increased recipient mortality [29].

\section{Preventing Transmission of Infections}

Donors are routinely screened for the presence and risk of infection. Donor screening for infection includes a medical and social history, serologic testing, blood and urine cultures, and chest X-ray (Table 1). The Organ Procurement and Transplantation Network (OPTN) policy requires donor screening for HIV, hepatitis B, hepatitis C, syphilis, cytomegalovirus, and Epstein-Barr virus. In the past, HTLV-1/2 testing was required but has been eliminated. The transmission of $\mathrm{HIV}, \mathrm{HBV}$, and $\mathrm{HCV}$ remains a concern despite the low risk. Donors may be deemed high risk for transmitting infectious disease based on epidemiology, behavior, and exposure [2]. Informed consent must be obtained and documented if a recipient accepts a donor at high risk of transmissible disease.

\section{Donor Risk Scores}

Donor risk scores are used as a tool to assess both donor organ acceptance as well as short-term mortality. Using the Eurotransplant Registry, Smits and colleagues created a heart transplant donor score using over 20 donor risk factors. Using a multivariate logistic regression model, they were able to assess the effects of donor factors on the discard rate. The heart donor score was significantly associated with acceptance of the donor organ as well as 3-year survival [30]. Weiss and colleagues developed a quantitative donor risk index using a 15-point scoring system that used four variables: ischemic time, donor age, race mismatching, and blood urea nitrogen/ creatinine ratio. Using this scoring system, each 1-point increase was associated with a 9-13\% increase risk of 1-year death [31]. Other risk prediction models such as IMPACT have more focus on recipient risk factors to develop a risk score. The 50-point scoring system was associated with increased odds of 1-year mortality [32] as well as 30-day and 5 -year mortality on a follow-up validation study [33].

Primary graft failure (PGF) remains one of the most common reasons for death within 30 days after orthotopic heart transplantation (OHT) [34]. Segovia and colleagues retrospectively reviewed a series of 621 heart transplants done at a single-center hospital in years between 1984 and 2006. PGF occurred in 56 out of 621 heart transplants. Using a multivariate analysis, six independent predictors were identified: $R$ ight 
Table 1 CCDT-recommended donor management goals

\begin{tabular}{ll}
\hline Mean arterial pressure & $60-100 \mathrm{mmHg}$ \\
Central venous pressure & $4-10 \mathrm{mmHg}$ \\
Left ventricular ejection fraction & $>50 \%$ \\
Pressor & Low dose \\
Arterial blood gas & $\mathrm{pH} 7.3-7.45$ \\
$\mathrm{PAO}_{2}: \mathrm{FIO}_{2}$ & $>300\left(\mathrm{on} \mathrm{PEEP}=5 \mathrm{~cm} \mathrm{H} \mathrm{H}_{2} \mathrm{O}\right)$ \\
Serum sodium & $135-160 \mathrm{mEq} / \mathrm{L}$ \\
Blood glucose & $<150 \mathrm{mg} / \mathrm{dL}$ \\
Hemoglobin & $>10 \mathrm{mg} / \mathrm{dL}$ \\
Urine output & $1-3 \mathrm{~mL} / \mathrm{kg} / \mathrm{h}$ \\
\hline
\end{tabular}

atrial pressure $\geq 10 \mathrm{mmHg}$, recipient $A$ ge $\geq 60$ years, Diabetes mellitus, Inotrope dependence, donor $A$ ge $\geq 30$ years, and Length of ischemic time $\geq 240 \mathrm{~min}$. Based on these results, a risk calculator, RADIAL, was developed. One point is assigned per risk for a total of 6 . Increasing RADIAL score was associated with significantly increased risk of PGF; a score of 4-6 was associated with $>5$-fold increase in risk of PGF $(\mathrm{OR}=5.33, p=0.01)$ [35].

\section{Donor Management}

\section{General}

Optimal cardiac donor management is a critical component to ensuring good graft function and the function of other organs. Brain death is associated with catecholamine surge and inflammatory activation [36]. This sympathetic storm results in intense peripheral vasoconstriction, coronary vasoconstriction, and hypertension. Cardiac dysfunction may ensue, and contraction band necrosis is a common pathologic finding $[37,38]$. An echo obtained within the first $6 \mathrm{~h}$ may show depressed cardiac function; however, since ejection fraction may improve after resuscitation, it may be better to avoid early echocardiograms in favor of waiting 6-12 h until physiologic derangements and electrolyte abnormalities have been corrected. An ejection fraction of $50 \%$ or greater is typically considered acceptable.

If there is loss of spontaneous circulation or CPR, ischemia time becomes a major concern. In this setting, vasoactives may be required to support circulation but they can negatively impact cardiac function and transplantability of the organ. Because of the physiologic derangements associated with brain death, circulatory support, restoration of organ perfusion, and correction of electrolyte abnormalities constitute early management. Donor management is typically performed in an intensive care setting, although specialized centers for managing donors have become more common. Central venous and arterial line placement and hemodynamic monitoring are recommended. In 2006, the Canadian Council for Donation and Transplantation (CCDT) published recommendations for donor management goals (DMGs) [39] (Table 1). Achieving optimal donor management goals has been demonstrated to increase the yield of organs per donor and transplants per donor [40]. Additional studies have shown an increased number of organs transplanted per donor when achieving greater than or equal to seven DMGs [40, 41].

Donors may have low levels of triiodothyronine $\left(\mathrm{T}_{3}\right)$ and thyroxin $\left(\mathrm{T}_{4}\right)$. As a result, thyroid hormone administration is frequently a component of aggressive management protocols in order to stabilize hemodynamics and to facilitate weaning of inotropes, although this practice is debated. In general, randomized trials have not demonstrated benefit with thyroid hormone alone or in combination with other hormone replacements while retrospective studies reported a beneficial effect [42]. Thyroid hormone does not appear to have any benefit on donor cardiac index or vasoactive drug requirement. Posterior pituitary function is also commonly affected leading to diabetes insipidus and associated fluid and electrolyte shifts. Additional hormone replacement may include corticosteroid and vasopressin [43-46].

\section{Perioperative and Surgical Considerations}

Primary allograft failure leads to catastrophic consequences and is among the leading causes of in-hospital mortality after cardiac transplantation; therefore, optimal myocardial protection plays a pivotal role in the success of the surgical procedure. While ex vivo perfusion systems may hold a promising role in the future, particularly within the donation after cardiocirculatory death donor population, the optimal technique of preserving myocardial viability and decreasing ischemia/reperfusion injury has not been established. Current organ preservation strategies are based on hypothermia in conjunction with a preservation solution, generally classified on the basis of sodium concentration as either extracellular or intracellular. The best solution for preservation of the donor heart has been a subject of debate and controversy, leading to a lack of standardization among transplant centers around the globe. A recent survey of the transplant centers in the USA revealed that at least 167 different solutions are utilized [47]. It appears that intracellular solutions (sodium content $<70 \mathrm{mEq} / \mathrm{L}$ ) are associated with a reduced risk of inhospital death. Cannata and colleagues studied the effect of three different myocardial preservation solutions (HTKCustodiol, Celsior, and St. Thomas) on intraoperative graft failure and in-hospital mortality. They observed no 
significant effect of the kinds of cardioplegic solution but did note that the recipient and donor age above 60 years and previous cardiac surgery were independent risk factors for in-hospital mortality, with odds ratios of 27.9 and 13.0, respectively [48]. Among the newer preservation solutions, Celsior $^{\circledR}$ is perhaps emerging as the solution of choice. Compared to HTK and University of Wisconsin solutions, Celsior may be associated with better post-transplant heart recovery and lower rates of graft failure $[49,50]$. Stahel et al. examined the benefits of an additional dose of cardioplegia dispensed immediately before implantation and found that it may benefit the organs suffering from longer ischemic times and improve early and late outcomes following transplantation [51].

Intraoperative blood loss secondary to coagulopathy and surgical bleeding has been associated with increased use of blood products, consequently leading to adverse outcomes following cardiac surgical procedures in general. An increasing proportion of heart transplant recipients in the USA are supported with ventricular assist devices and are receiving anticoagulation. Some require re-do sternotomy and extensive mediastinal dissection, leading to increased blood loss and utilization of blood products. Awad and colleagues investigated the effect of prior sternotomy on the postoperative mortality and morbidity after heart transplantation. While they demonstrated no difference in 60-day mortality, the 1-year survival was higher in the primary sternotomy group. The patients with prior operation had longer cardiopulmonary bypass times and ICU and hospital stays and required more blood products. The subgroup of patients with prior VAD implantation had lower 60-day and 1-year survival [52]. Historically, anticoagulation with warfarin has been discontinued or rapidly reversed immediately prior to transplantation. A recent retrospective data analysis by Morris et al. found no correlation between preoperative or postoperative international normalized ratio (INR) and chest tube output. They also noted no correlation between the preoperative INR and the perioperative use of fresh frozen plasma, suggesting that preoperative warfarin may be safely continued in patients awaiting transplantation [53].

\section{The Future of Donor Selection and Management}

\section{Donor Selection}

Evidence-based donor selection remains a challenge. The Donor Heart Study, an NIH-funded trial, is investigating evidence-based evaluation and acceptance of donor hearts. The goals of this study are to collect systematic data on cardiac function and real-time data on reasons for donor non-acceptance and to develop clinical tools for decision-making.

\section{Ex Vivo Perfusion}

The major limitations to increasing the donor pool are distance and the subsequent risk of prolonged ischemic time. The transportable Organ Care System (TranMedics; Andover, MA, USA) is a clinical ex vivo heart perfusion platform that can maintain the donor heart in a warm, beating, near-physiological state for transplantation. The Organ Care System is the only clinical platform for ex vivo perfusion of donor hearts. The results of the PROCEED II study, which randomized 130 patients to the Organ Care system, were recently published and demonstrated noninferiority of the Organ Care System when compared to standard cold storage on short-term clinical outcomes [54]. Additional studies are needed to determine the best application of ex vivo perfusion. This technology may decrease the consequences of ischemic time and allow procurement of organs remote to the transplant site in the future [54].

\section{Donation After Circulatory Death}

Donation after circulatory death (DCD) was the method used on the first heart transplant in 1967 by Christiaan Barnard [55]. Since that time, the definition of brain death has been introduced, leading to the more common method of donation after brain death (DBD) transplantation. Recently, there has been increasing interest in DCD as a method to increase donor organ availability [56]. Previous studies have shown similar outcomes at 1 year for DCD kidney [57] and lung [58] transplants when compared to transplants donated after brain death. Factors that complicate DCD transplantation in heart transplantation include the inability to assess cardiac function and the risk of warm ischemic insult [59]. Recently, a case series was published of three DCD heart transplantations. Four hearts were retrieved and transferred to an Organ Care System for preservation, resuscitation, and transportation; three of the hearts were transplanted. Two of the recipients required temporary mechanical circulatory support, but all three had normal cardiac function within 1 week. Clearly, additional evaluation of DCD in heart transplant is required; however, this may represent an additional way to expand the donor pool in the future.

\section{Conclusion}

Donor selection and management are the initial determinants of outcomes after heart transplant. Systematic evaluation of risk factors and standardized donor management will provide 
the basis for optimal use of donor hearts. Evidence-based donor selection and management are needed and may serve to broaden the donor pool.

\section{Compliance with Ethics Guidelines}

Conflict of Interest Dr. Gillian Grafton, Dr. Gordon Samoukovic, and Dr. Monica Colvin declare that they have no conflict of interest.

Human and Animal Rights and Informed Consent This article does not contain any studies with human or animal subjects performed by any of the authors.

\section{References}

1. Kilic A, Weiss ES, Yuh DD, Shah AS, Cameron DE, Baumgartner WA, et al. Institutional factors beyond procedural volume significantly impact center variability in outcomes after orthotopic heart transplantation. Ann Surg. 2012;256(4):616-23.

2. Russo MJ, Chen JM, Sorabella RA, Martens TP, Garrido M, Davies $\mathrm{RR}$, et al. The effect of ischemic time on survival after heart transplantation varies by donor age: an analysis of the United Network for Organ Sharing database. J Thorac Cardiovasc Surg. 2007;133(2):554-9.

3. John R. Donor management and selection for heart transplantation. Semin Thorac Cardiovasc Surg. 2004;16(4):364-9.

4. Khush KK, Menza RL, Babcock WD, Zaroff JG. Donor cardiac troponin I levels do not predict recipient survival after cardiac transplantation. J Heart Lung Transplant. 2007;26(10):1048-53.

5. Hashimoto S, Kato TS, Komamura K, Hanatani A, Niwaya K, Funatsu T, et al. Utility of echocardiographic evaluation of donor hearts upon the organ procurement for heart transplantation. $\mathrm{J}$ Cardiol. 2011;57(2):215-22.

6. Kuppahally SS, Valantine HA, Weisshaar D, Parekh H, Hung YY, Haddad F, et al. Outcome in cardiac recipients of donor hearts with increased left ventricular wall thickness. Am J Transplant. 2007;7(10):2388-95.

7. Gao HZ, Hunt SA, Alderman EL, Liang D, Yeung AC, Schroeder JS. Relation of donor age and preexisting coronary artery disease on angiography and intracoronary ultrasound to later development of accelerated allograft coronary artery disease. J Am Coll Cardiol. 1997;29(3):623-9.

8. Li H, Tanaka K, Anzai H, Oeser B, Lai D, Kobashigawa JA, et al. Influence of pre-existing donor atherosclerosis on the development of cardiac allograft vasculopathy and outcomes in heart transplant recipients. J Am Coll Cardiol. 2006;47(12):2470-6.

9. Benden C, Goldfarb SB, Edwards LB, Kucheryavaya AY, Christie JD, Dipchand AI, et al. The registry of the International Society for Heart and Lung Transplantation: seventeenth official pediatric lung and heart-lung transplantation report-2014; focus theme: retransplantation. J Heart Lung Transplant. 2014;33(10):1025-33.

10. Del Rizzo DF, Menkis AH, Pflugfelder PW, Novick RJ, McKenzie FN, Boyd WD, et al. The role of donor age and ischemic time on survival following orthotopic heart transplantation. J Heart Lung Transplant. 1999;18(4):310-9.

11. Roig E, Almenar L, Crespo-Leiro M, Segovia J, Mirabet S, Delgado J, et al. Heart transplantation using allografts from older donors: multicenter study results. J Heart and Lung Transplant. 2015;34:790-6.
12. Prieto D, Correia P, Baptista M, Antunes MJ. Outcome after heart transplantation from older donor age: expanding the donor pool. Eur J Cardio-Thorac Surg. 2015;47(4):672-8.

13. Tehrani YS, Yu Z, Luu M, Liou F, Rafiei M, Hamilton M, et al. The policy of placing older donors into older recipients: is it worth the risk? Clin Transpl. 2014;28(7):802-7.

14. Lietz K, John R, Mancini DM, Edwards NM. Outcomes in cardiac transplant recipients using allografts from older donors versus mortality on the transplant waiting list: implications for donor selection criteria. J Am Coll Cardiol. 2004;43(9):1553-61.

15. Prendergast TW, Furukawa S, Beyer 3rd AJ, Browne BJ, Eisen HJ, Jeevanandam V. The role of gender in heart transplantation. Ann Thorac Surg. 1998;65(1):88-94.

16. McCarthy JF, McCarthy PM, Massad MG, Cook DJ, Smedira NG, Kasirajan V, et al. Risk factors for death after heart transplantation: does a single-center experience correlate with multicenter registries? Ann Thorac Surg. 1998;65(6):1574-8. discussion 8-9.

17. Solomon NA, McGiven JR, Alison PM, Ruygrok PN, Haydock DA, Coverdale HA, et al. Changing donor and recipient demographics in a heart transplantation program: influence on early outcome. Ann Thorac Surg. 2004;77(6):2096-102.

18. Tsai FC, Marelli D, Bresson J, Gjertson D, Kermani R, Ardehali A, et al. Recent trends in early outcome of adult patients after heart transplantation: a single-institution review of 251 transplants using standard donor organs. Am J Transplant. 2002;2(6):539-45.

19. Stehlik J, Feldman DS, Brown RN, VanBakel AB, Russel SD, Ewald GA, et al. Interactions among donor characteristics influence post-transplant survival: a multi-institutional analysis. J Heart Lung Transplant. 2010;29(3):291-8.

20. Khush KK, Kubo JT, Desai M. Influence of donor and recipient sex mismatch on heart transplant outcomes: analysis of the International Society for Heart and Lung Transplantation Registry. J Heart Lung Transplant. 2012;31(5):459-66.

21. Al-Khaldi A, Oyer PE, Robbins RC. Outcome analysis of donor gender in heart transplantation. J Heart Lung Transplant. 2006;25(4):461-8.

22. Weiss ES, Allen JG, Patel ND, Russell SD, Baumgartner WA, Shah AS, et al. The impact of donor-recipient sex matching on survival after orthotopic heart transplantation: analysis of 18000 transplants in the modern era. Cir Heart Fail. 2009;2(5):401-8.

23. Reed RM, Netzer G, Hunsicker L, Mitchell BD, Rajagopal K, Scharf S, et al. Cardiac size and sex-matching in heart transplantation: size matters in matters of sex and the heart. JACC Heart Failure. 2014;2(1):73-83.

24. Taylor DO, Edwards LB, Boucek MM, Trulock EP, Aurora P, Christie J, et al. Registry of the International Society for Heart and Lung Transplantation: twenty-fourth official adult heart transplant report-2007. J heart Lung Transplant. 2007;26(8):769-81.

25. Patel ND, Weiss ES, Nwakanma LU, Russell SD, Baumgartner WA, Shah AS, et al. Impact of donor-to-recipient weight ratio on survival after heart transplantation: analysis of the United Network for Organ Sharing Database. Circulation. 2008;118(14 Suppl):S838.

26. Goland S, Czer LS, Kass RM, De Robertis MA, Mirocha J, Coleman B, et al. Pre-existing pulmonary hypertension in patients with end-stage heart failure: impact on clinical outcome and hemodynamic follow-up after orthotopic heart transplantation. J Heart Lung Transplant. 2007;26(4):312-8.

27. Kwon MH, Wong S, Kittleson M, Ardehali A, Laks H, Shemin RJ, et al. Selecting oversized donor cardiac allografts for patients with pulmonary hypertension may be unnecessary. Transplant Proc. 2014;46(5):1497-501.

28. Taylor DO, Edwards LB, Boucek MM, Trulock EP, Keck BM, Hertz MI. The Registry of the International Society for Heart and Lung Transplantation: twenty-first official adult heart transplant report-2004. J Heart Lung Transplant. 2004;23(7):796-803. 
29. Khush KK, Menza R, Nguyen J, Zaroff JG, Goldstein BA. Donor predictors of allograft use and recipient outcomes after heart transplantation. Cir Heart Fail. 2013;6(2):300-9.

30. Smits JM, De Pauw M, de Vries E, Rahmel A, Meiser B, Laufer G, et al. Donor scoring system for heart transplantation and the impact on patient survival. J Heart Lung Transplant. 2012;31(4):387-97.

31. Weiss ES, Allen JG, Kilic A, Russell SD, Baumgartner WA, Conte $\mathrm{JV}$, et al. Development of a quantitative donor risk index to predict short-term mortality in orthotopic heart transplantation. J Heart Lung Transplant. 2012;31(3):266-73.

32. Weiss ES, Allen JG, Arnaoutakis GJ, George TJ, Russell SD, Shah AS, et al. Creation of a quantitative recipient risk index for mortality prediction after cardiac transplantation (IMPACT). Ann Thorac Surg. 2011;92(3):914-21. discussion 21-2.

33. Kilic A, Allen JG, Weiss ES. Validation of the United Statesderived Index for Mortality Prediction After Cardiac Transplantation (IMPACT) using international registry data. J Heart Lung Transplant. 2013;32(5):492-8.

34. Stehlik J, Edwards LB, Kucheryavaya AY, Benden C, Christie JD, Dipchand AI, et al. The Registry of the International Society for Heart and Lung Transplantation: 29th official adult heart transplant report-2012. J Heart Lung Transplant. 2012;31(10):1052-64.

35. Segovia J, Cosio MD, Barcelo JM, Bueno MG, Pavia PG, Burgos $\mathrm{R}$, et al. RADIAL: a novel primary graft failure risk score in heart transplantation. J Heart Lung Transplant. 2011;30(6):644-51.

36. Powner DJ, Hendrich A, Nyhuis A, Strate R. Changes in serum catecholamine levels in patients who are brain dead. J Heart Lung Transplant. 1991;11(6):1046-53.

37. Dujardin KS, McCully RB, Wijdicks EF, Tazelaar HD, Seward JB, McGregor CG, et al. Myocardial dysfunction associated with brain death: clinical, echocardiographic, and pathologic features. J heart Lung Transplant. 2001;20(3):350-7.

38. Pratschke J, Wilhelm M, Kusaka M, Basker M, Cooper D, Hancock $\mathrm{W}$, et al. Brain death and its influence on donor organ quality and outcome after transplantation. Transplantation. 1999;67(3):343-8.

39. Shemie SD, Ross H, Pagliarello J, Baker AJ, Greig PD, Brand T, et al. Organ donor management in Canada: recommendations of the forum on Medical Management to Optimize Donor Organ Potential. CMAJ. 2006;174(6):S13-32.

40. Patel MS, Zatarain J, De La Cruz S, Sally MB, Ewing T, Crutchfield $\mathrm{M}$, et al. The impact of meeting donor management goals on the number of organs transplanted per expanded criteria donor: a prospective study from the UNOS Region 5 Donor Management Goals Workgroup. JAMA Surg. 2014;149(9):96975 .

41. Malinoski DJ, Daly MC, Patel MS, Oley-Graybill C, Foster 3rd CE, Salim A. Achieving donor management goals before deceased donor procurement is associated with more organs transplanted per donor. J Trauma. 2011;71(4):990-5. discussion 6.

42. Macdonald PS, Aneman A, Bhonagiri D, Jones D, O'Callaghan G, Silvester W, et al. A systematic review and meta-analysis of clinical trials of thyroid hormone administration to brain dead potential organ donors. Crit Care Med. 2012;40(5):1635-44.

43. Rosendale JD, Kauffman HM, McBride MA, Chabalewski FL, Zaroff JG, Garrity ER, et al. Aggressive pharmacologic donor management results in more transplanted organs. Transplantation. $2003 ; 75(4): 482-7$.
44. Rosendale JD, Kauffman HM, McBride MA, Chabalewski FL, Zaroff JG, Garrity ER, et al. Hormonal resuscitation yields more transplanted hearts, with improved early function. Transplantation. 2003;75(8):1336-41.

45. Venkateswaran RV, Steeds RP, Quinn DW, Nightingale P, Wilson IC, Mascaro JG, et al. The haemodynamic effects of adjunctive hormone therapy in potential heart donors: a prospective randomized double-blind factorially designed controlled trial. Eur Heart J. 2009;30(14):1771-80.

46. Nath DS, Ilias Basha H, Liu MH, Moazami N, Ewald GA. Increased recovery of thoracic organs after hormonal resuscitation therapy. J Heart Lung Transplant. 2010;29(5):594-6.

47. Demmy TL, Biddle JS, Bennett LE, Walls JT, Schmaltz RA, Curtis JJ. Organ preservation solutions in heart transplantation - patterns of usage and related survival. Transplantation. 1997;63(2):262-9.

48. Cannata A, Botta L, Colombo T, Russo CF, Taglieri C, Bruschi G, et al. Does the cardioplegic solution have an effect on early outcomes following heart transplantation? Eur J Cardio-Thorac Surg. 2012;41(4):e48-52. discussion e-3.

49. Garlicki M. May preservation solution affect the incidence of graft vasculopathy in transplanted heart? Annals Transplant Q Pol Transplant Soc. 2003;8(1):19-24.

50. Hernandez A, Borrego JM, Gomez S, Gutierrez E, Lage E, Hinojosa R, et al. Myocardial preservation using Celsior: clinical results in high-risk cardiac transplantation. Transplant Proc. 2005;37(3):1543-5.

51. Tevaearai Stahel HT, Unger D, Schmidli J, Gahl B, Englberger L, Kadner A, et al. Supplemental cardioplegia immediately before graft implantation may improve early post-transplantation outcome. Frontiers Surgery. 2014;1:46.

52. Awad M, Czer LS, Mirocha J, Ruzza A, Rafiei M, Reich H, et al. Prior sternotomy increases the mortality and morbidity of adult heart transplantation. Transplant Proc. 2015;47(2):485-97.

53. Morris CD, Vega JD, Levy JH, Buist NN, Smith AL, Despotis GJ, et al. Warfarin therapy does not increase bleeding in patients undergoing heart transplantation. Ann Thorac Surg. 2001;72(3):714-8.

54. Ardehali A, Esmailian F, Deng M, Soltesz E, Hsich E, Naka Y, et al. Ex-vivo perfusion of donor hearts for human heart transplantation (PROCEED II): a prospective, open-label, multicentre, randomised non-inferiority trial. Lancet. 2015

55. Barnard $\mathrm{CN}$. The operation. A human cardiac transplant: an interim report of a successful operation performed at Groote Schuur Hospital, Cape Town. S Afr Med J. 1967;41(48):1271-4.

56. Zaroff JG, Rosengard BR, Armstrong WF, Babcock WD, D'Alessandro A, Dec GW, et al. Consensus conference report: maximizing use of organs recovered from the cadaver donor: cardiac recommendations, March 28-29, 2001, Crystal City, Va. Circulation. 2002;106(7):836-41.

57. Snoeijs MG, Schaubel DE, Hene R, Hoitsma AJ, Idu MM, Ijzermans JN, et al. Kidneys from donors after cardiac death provide survival benefit. J Am Soc Nephrol. 2010;21(6):1015-21.

58. Machuca TN, Mercier O, Collaud S, Tikkanen J, Krueger T, Yeung $\mathrm{JC}$, et al. Lung transplantation with donation after circulatory determination of death donors and the impact of ex vivo lung perfusion. Am J Transplant. 2015;15(4):993-1002.

59. Manara AR, Murphy PG, O'Callaghan G. Donation after circulatory death. Br J Anaesth. 2012;108 Suppl 1:1108-21. 